This is an Open Access article distributed under the terms of the Creative Commons Attribution-Noncommercial License, which permits unrestricted use, distribution, and reproduction in any noncommercial medium, provided the original work is properly cited.

\title{
THE INTERSTELLAR GAS SEEN IN THE MID- AND FAR-INFRARED: THE PROMISE OF SPICA SPACE TELESCOPE
}

\author{
Javier R. Goicoechea ${ }^{1}$ and José Cernicharo ${ }^{1,2}$ \\ ${ }^{1}$ Centro de Astrobiología, CSIC-INTA, Madrid, Spain \\ ${ }^{2}$ Herschel Space Observatory Mission Scientist
}

\begin{abstract}
The mid- and far-IR spectral ranges are critical windows to characterize the physical and chemical processes that transform the interstellar gas and dust into stars and planets. Sources in the earliest phases of star formation and in the latest stages of stellar evolution release most of their energy at these wavelengths. Besides, the mid- and far-IR ranges provide key spectral diagnostics of the gas chemistry (water, light hydrides, organic species ...), of the prevailing physical conditions $\left(\mathrm{H}_{2}\right.$, atomic fine structure lines ...), and of the dust mineral and ice composition that can not be observed from ground-based telescopes. With the launch of JAXA's SPICA telescope, uninterrupted studies in the mid- and far-IR will be possible since ESA's Infrared Space Observatory (1995). In particular, SAFARI will provide full access to the $\sim 34-210 \mu \mathrm{m}$ waveband through several detector arrays and flexible observing modes (from broadband photometry to medium resolution spectroscopy with $R \sim 3,000$ at $63 \mu \mathrm{m}$ ), and reaching very high line sensitivities $\left(\sim 10^{-19} \mathrm{~W} \mathrm{~m}^{-2}, 5 \sigma-1 \mathrm{hr}\right)$ within a large FOV $\left(\sim 2^{\prime} \times 2^{\prime}\right)$. Compared to previous farIR instruments (ISO/LWS, AKARI/FIS, Spitzer/MIPS and Herschel/PACS), SAFARI will provide a superior way to obtain fully-sampled spectro-images and continuous SED of very faint and extended ISM sources in a wavelength domain not accessible to JWST or ALMA. The much increased sensitivity of SPICA will allow us to step forward and reveal not only the chemical complexity in the local ISM, but also in the extragalactic ISM routinely.
\end{abstract}

Key words: astrochemistry - infrared: ISM - ISM: clouds - line: formation - Missions: SPICA

\section{INTRODUCTION}

Due to the atmospheric opacity, the mid- and far-infrared domains have been the latest spectral windows used in astrochemistry. The potential of opening a new spectral wavelength range for molecular spectroscopy begun to be fully exploited by ESA's Infrared Space Observatory, ISO (Kessler et al. 1996), it has been successfully continued by NASA's Spitzer (Werner et al. 2004) and JAXA's AKARI (Murakami et al. 2007), and it will further advanced with ESA's Herschel Space Observatory (Pilbratt et al. 2009).
Before ISO, the mid- and far-IR spectra of the most relevant interstellar (ISM) and circumstellar (CSM) sources were unknown. In particular, most gas phase species emitting in the mid- and far-IR were unidentified. Nowadays we know that the spectral features that can be detected in this domain are essential to study the physical conditions and the chemical content of a broad range of astronomical environments. Such diagnostics include atomic fine structure lines, $\mathrm{PAH}$ bands, $\mathrm{H}_{2}$ lines, light hydrides and many non-polar molecules that can not be detected with ground-based radio telescopes (e.g., PdBI, SMA or ALMA and SKA in the future). Besides, the mid- and far-IR provide access to a great variety of ice and mineral features that help us to understand the life-cycle of cosmic dust.

In this short contribution we argue why a very sensitive space telescope covering the full mid- and far-IR range can decisively contribute to improve our understanding of the chemical complexity in the Universe. We also review some representative examples of previous mid- and far-IR observations towards very bright ISM and CSM sources in the Milky Way. These spectra remain unique examples of the diagnostic power of this domain, and are archetypes of what much more sensitive space telescopes (SPICA!) will routinely observe in much fainter regions of the sky, and that are beyond the sensitivity capabilities of Herschel. The contribution of dust grains and PAHs to the mid- and far-IR spectrum of different environments is well covered by other papers in these proceedings. Here we shall put more emphasis on gas phase molecular spectroscopy. More detailed reviews can be found in e.g., van Dishoeck (2004) or Cernicharo \& Crovisier (2005).

\section{WHY ANOTHER MID- AND FAR-IR SPACE TELESCOPE?}

The life-cycle of interstellar gas and dust has been traditionally studied through line absorption observations in the UV and visible domains (e.g., the diffuse and translucent ISM) and through submm, millimeter and centimeter observations with ground-based radiotelescopes (usually by detecting emission lines). Due to the atmospheric opacity and because of the nature of IR detectors, observations over the entire mid- and far-IR bands require space telescopes and cryogenic instrumentation (down to a few $\mathrm{mK}$ !). Both requirements imply a significant technological (and financial) challenge, as well as a notable international collaboration. All of them can be better justified if: 


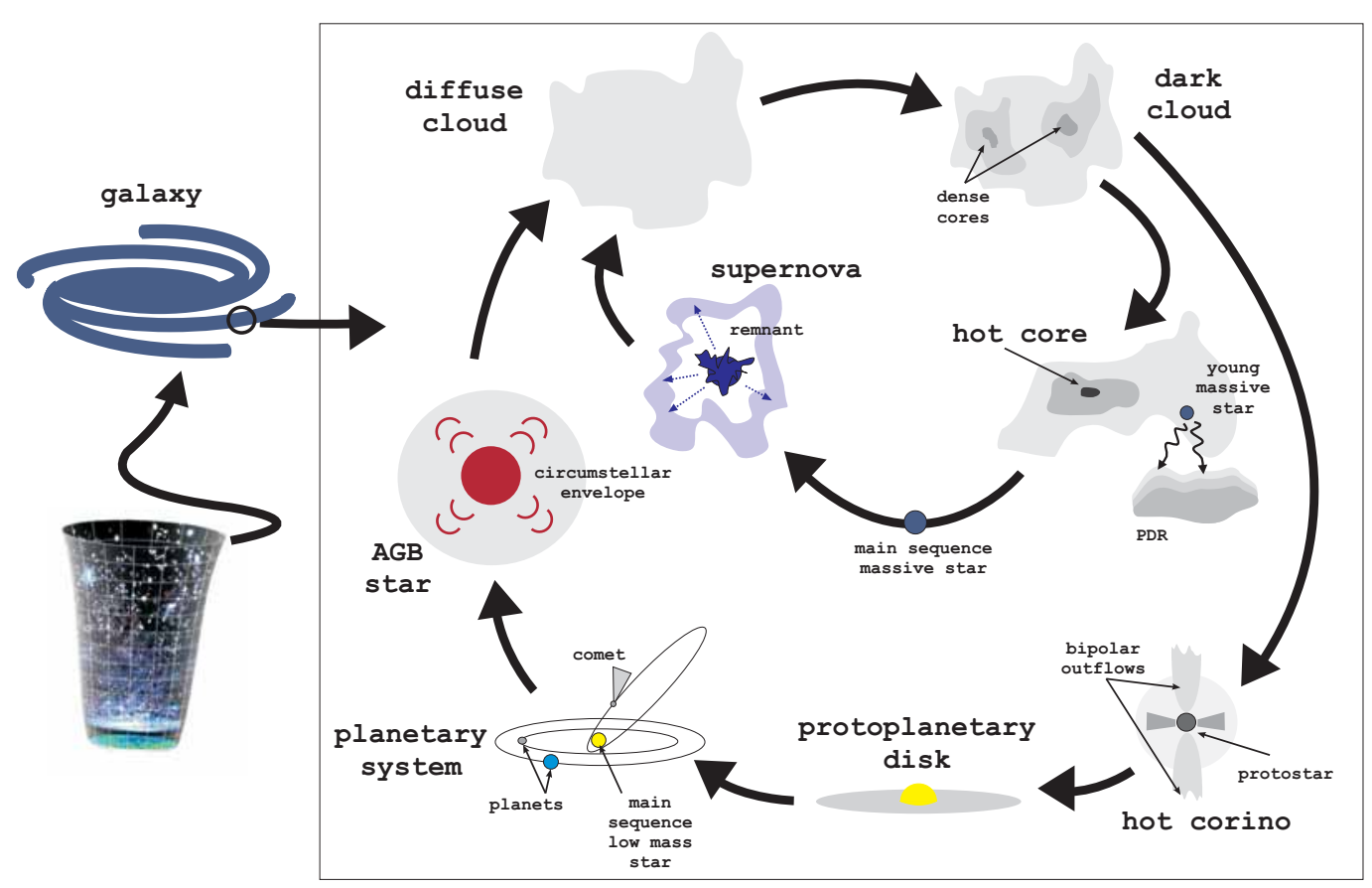

Figure 1. Gas and dust life-cycle in the Molecular Universe. The life-cycle of interstellar matter is tied to stellar evolution and the formation of planetary systems. SPICA will follow the gas, mineral and ice inventory, as well as their formation, processing and destruction mechanisms by targeting all relevant environments in the Milky Way and in external galaxies. Agúndez (2009).

1) Science drivers are exciting and unique (hard to achieve with other instrumentation). 2) Instruments provide outstanding scientific results (with great impact in the field).

In (gas phase) astrochemistry there are three particular areas that are only accessible in the mid- and far-IR:

Fine structure lines from key gas reservoirs such as [Fe II]26, [Si II]35, [O I]63,145 and [C II] $158 \mu \mathrm{m}\left(E_{i}<13.6 \mathrm{eV}\right)$ that are critical diagnostics of the neutral gas component of the ISM and are often the most important cooling lines. On the other hand, fine structure lines of ions with $E_{i}>13.6 \mathrm{eV}([\mathrm{O}$ III $] 52,88,[\mathrm{~N}$ III $] 57 \mu \mathrm{m}, \ldots)$ trace the ionized gas component (e.g., H II regions: electron densities, ionizing radiation strength, etc.). All these lines are usually bright and are not affected by extinction problems as they are at shorter wavelengths (visible and near-IR).

Light molecules that have their pure rotational transitions in this range: e.g., $\mathrm{H}_{2}$, water vapor, $\mathrm{OH}, \mathrm{HD}, \mathrm{H}_{2} \mathrm{D}^{+}$ and many other hydrides. Together with the above atomic fine structure lines, their spectral signature allows one to penetrate heavily obscured regions like star forming regions or the ISM in galactic nuclei, and provides means to derive the physical conditions (densities, temperatures, UV fields) and the chemical composition in harsh environments (e.g., UV irradiated regions, XDRs, shockedregions, etc.). Heavier species $(\mathrm{CO}, \mathrm{HCN}, \ldots)$ have their high energy transitions in this domain (their "high- $J$ lines") and trace even higher temperature and density phenomena (e.g., strongly shocked-regions such as SNe remnants, the inner circumstellar shells around evolved stars, etc.).
Organic chemistry: symmetrical molecules (i.e., all species lacking a permanent electric dipole) do not have pure rotational lines (i.e., to be observed from radiotelescopes) but do show vibration-rotation transitions in the mid- and far-IR (stretching and bending modes). Very relevant and abundant species of this kind are, for example, acetylene $\left(\mathrm{C}_{2} \mathrm{H}_{2}\right)$, methane $\left(\mathrm{CH}_{4}\right)$, carbon dioxide $\left(\mathrm{CO}_{2}\right)$, benzene $\left(\mathrm{C}_{6} \mathrm{H}_{6}\right)$, many different pure carbon chains $\left(\mathrm{C}_{n}\right)$ and of course, the ubiquitous polycyclic aromatic hydrocarbons (PAHs).

\section{The INTERSTELlaR GAS AND DUST LIFE-CYCLE}

The first of the four Cosmic Vision (CV) Themes proposed by ESA, "What are the conditions for planet formation and the emergence of life?" calls for a mission able to "investigate star-formation areas and protostars..." and "investigate the conditions for star formation and evolution". As stated in the CV objectives, "we still lack a comprehensive theory explaining why and how stars form from interstellar matter and, apparently quite often, planetary systems with them". SPICA instruments will make decisive contributions to the study of the physical and chemical processes that transform the "gas and dust into stars and planets", or in other words, will follow the life-cycle of matter: from the formation of dust grains in circumstellar shells, to their incorporation to planet forming disks, some of them maybe similar to what our own Solar System looked like at the time of its formation (see the gas and dust life-cycle diagram in Figure 1). 
SPICA observations will allow us to significantly improve our knowledge of many areas related to planetary formation and evolution (see detailed contributions in these proceedings). As a unique complement, SPICA will decisively contribute to unveil the initial conditions for star formation (a mission able to "map the birth of stars and planets by peering into the highly obscured cocoons where they form") and in the processes associated with stellar death (evolved stars, supernova remnants, etc.) that both enrich and process the ISM gas and dust for a subsequent generation of star formation.

The process of molecular cloud formation, star formation and star death are poorly understood because of the large amounts of dust obscuring the molecular cloud sites where stars are born, as well as the circumstellar envelopes (CSE) of molecular gas and dust created at the end of Sun-like stars' life. Since the dust becomes "transparent" at far-IR wavelengths, this spectral window provides the natural way to penetrate inside these environments. By observing in the mid- and far-IR, SPICA will provide and unprecedented window into key aspects of the gas and dust life-cycle: from its formation in evolved stars, its evolution in the ISM, its processing in supernova-generated shock waves and near massive stars (winds, H II regions, etc.) to its final incorporation into star forming cores, protostars, protoplanetary disks and eventually planets.

Very important gas-phase species that can not be observed from the ground have their spectral signatures in the operative range of SPICA. Such spectral diagnostics provide clues on the elemental abundances of key elements $(\mathrm{C}, \mathrm{O}, \mathrm{D}, \mathrm{Si}, \ldots)$ and they also provide deep insights into the gas/dust chemical interplay: deuterium enrichment, ice formation, grain growth, evaporation, photodesorption or metal depletion on grains (e.g., Okada et al. 2008).

\section{LOCAL AND EXTRAGALACTIC ISM With SPICA}

An important fraction of the interactions that transform the gas and dust into dense molecular clouds and stars, takes place over very large scale regions, several arc minutes size (e.g. diffuse ISM clouds, cirrus, high latitude clouds, star forming regions, circumstellar shells, supernova remnants, etc.) that are too faint to be mapped entirely by Herschel in spectroscopy mode, or too extended to be mapped with ground-based interferometers. In this context, the niche of SPICA/SAFARI is to provide far-IR spectro-images (of many key spectral diagnostics simultaneously) of the regions that are too obscured for JWST to examine or too warm/extended to be efficiently traced with ALMA in the millimeter domain.

SPICA instruments have the appropriate combination of spatial and spectral resolution together with high sensitivity and large field-of-view (FOV) to follow the evolution of interstellar matter over very large spatial scales (e.g., by taking full SEDs and spectra of distant star forming regions), study meaningful samples of compact sources (e.g., hundreds of protostars, distant evolved stars and disks around all types of stars and ages) and out to large distances (inaccessible to previous telescopes).

In the following we just list several fields where we anticipate SAFARI can play a unique role (difficult to achieve with other telescopes):

- Spectro-imaginery of the ISM in nearby galaxies.

- Chemical complexity beyond our galaxy.

- Continuous SEDs of Young Stellar Objects.

- High-mass star formation in distant SF regions.

- Evolved stars and interaction with ISM.

- Supernova remnants (dust formation and processing?).

- Molecular cloud formation.

- The first identification of PAH molecules in space.

Thanks to its much improved sensitivity, SPICA will extend our knowledge of the physics and chemistry of the ISM matter in our galaxy to similar detailed studies of the ISM in nearby, spatially resolved galaxies (see e.g., González-Alfonso et al. 2004; Goicoechea et al. 2005).

\subsection{What Will not Be DONE By Herschel \& JWST?}

The successful launch of ESA's Herschel Space Observatory in May 2009 - the biggest IR telescope ever launched suggests that Herschel will be a major breakthrough for far-IR and submm astronomy. At the time of writing, commissioning has been declared successful and the instruments are now in the performance verification phase. However, Herschel has several constraints and there will be many critical areas of the ISM and CSM research that will not be covered due to limited sensitivity, wavelength coverage or spectral resolution:

- Herschel observes the $\sim 55$ to $672 \mu \mathrm{m}$ waveband so it does not access the mid-IR domain where most mineralogy and ice studies can be carried out. Similarly, it can not observe the mid-IR emission of PAHs and most organic molecules or the high excitation transitions (high- $J$ ) of many species. SPICA, however, will cover the full $\sim 5$ to $210 \mu \mathrm{m}$ waveband (baseline).

- Herschel is a "warm" telescope which greatly limits its sensitivity and thus mapping speed to obtain spectroimages of very extended/distant ISM sources. SAFARI, the far-IR imaging-spectrometer for SPICA, will cover the critical $\sim 34-210 \mu \mathrm{m}$ that is not accessible to JWST or ALMA with a factor $\sim 200$ (in photometry) and $\sim 20$ (in spectroscopy) better sensitivity than Herschel /PACS, and with a wider instantaneous FOV (i.e., $\sim 2^{\prime} \times 2^{\prime}$, a factor $\sim 6.5$ larger $)$.

- JWST/MIRI (>2014) will operate in the mid-IR $(\sim 5$ $28 \mu \mathrm{m}$ band), so it does not cover the far-IR. MIRI will provide imaging and medium resolution spectroscopy $(R \sim 3,000)$ over a small FOV (a few $\operatorname{arcsec}^{2}$ ). SPICA's mid-IR cameras and spectrometers will provide larger FOVs and out to an order of magnitude higher spectral resolution $(R \sim 30,000)$ in selected mid-IR wavebands. 

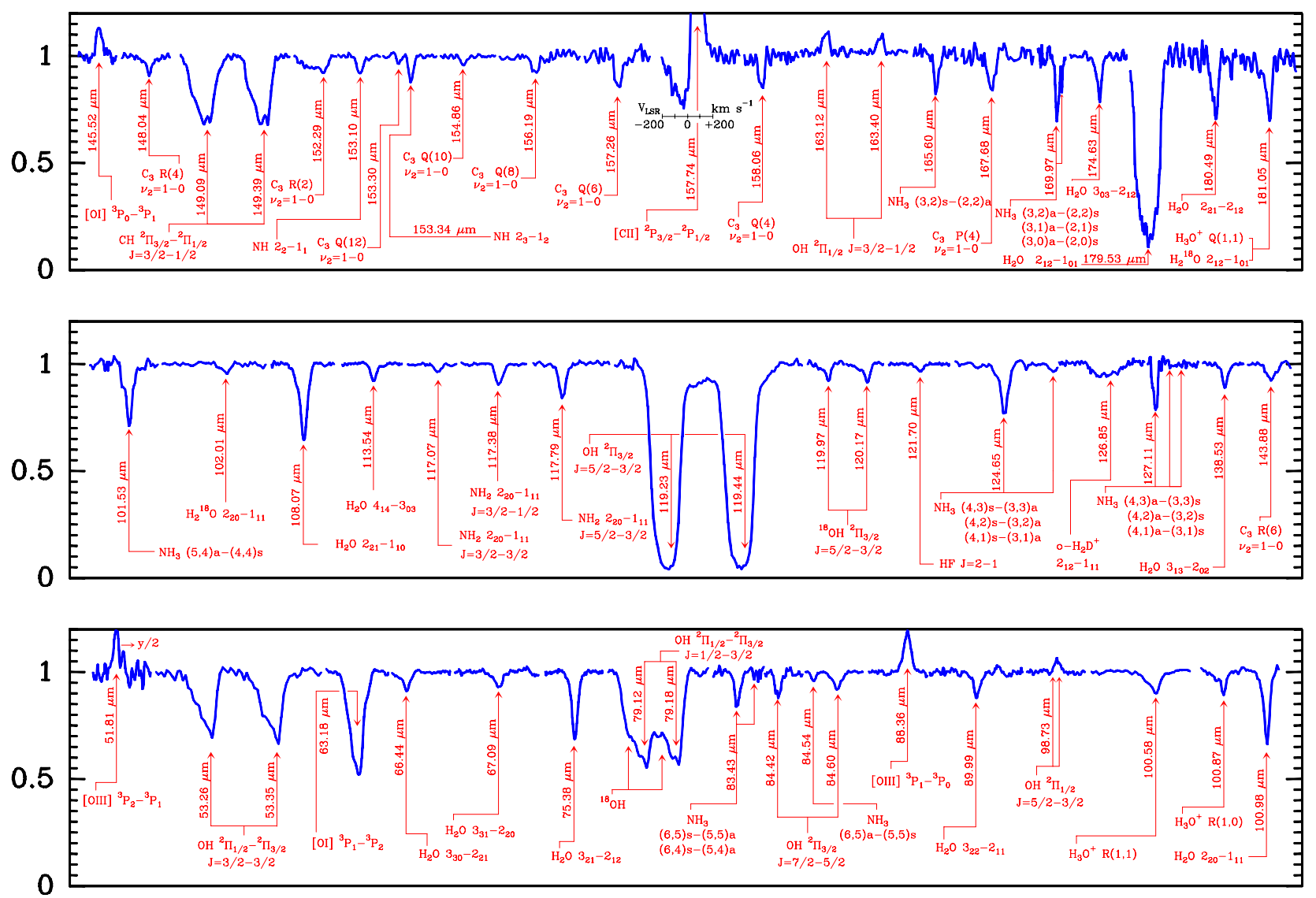

Figure 2. Far-IR spectrum of Sgr B2 star forming region in the Galactic Center taken with the ISO/LWS. Line detections have been discussed in detail in different works (see Goicoechea et al. 2004; Polehampton et al. 2007 and references therein).

Despite its modest monolithic mirror size ( $D \sim 3.5 \mathrm{~m}$, similar to Herschel) the SPICA telescope will be actively cooled down to $\sim 4.5 \mathrm{~K}$ (compared to $\sim 45 \mathrm{~K}$ through passive cooling for JWST), and thus will reach similar sensitivities than JWST/MIRI in the mid-IR (around $\sim 1 \mu \mathrm{Jy}$ ) but with a much increased wavelength range (including the far-IR!), wider FOV and increased observing efficiency due to the use of multiple detector arrays.

\subsection{What WiLl Not Be DONE By ALMA?}

The Atacama Large Millimeter Array (ALMA) will be fully operational the next decade. With its extremely high angular resolution and huge collecting area, ALMA will be a major revolution in many fields of the cold and molecular Universe. However, ALMA observes at wavelengths longer than $\sim 315 \mu \mathrm{m}(<950 \mathrm{GHz})$ and for practical reasons (it is a very long-baseline interferometer) it is not designed to carry out extensive surveys of very large regions of the sky. In addition, there are critical spectral diagnostics (at their restframe wavelengths) that are not accessible to ALMA:

- The study of the oxygen chemistry will be limited to observations of $\mathrm{CO}, \mathrm{HCO}^{+}$and other trace species, but it will not observe the emission of the major oxygen reservoirs (e.g., atomic oxygen, water ice, $\mathrm{CO}_{2}$ or the thermal line emission of water vapor).

- The most important atomic and ionic fine structure lines are outside the coverage of ALMA, which will limit the determination of the physical conditions (temperatures, densities and radiation fields) and the study of the energy balance (heating and cooling) in PDRs, XDRs, and shock-like environments.

- ALMA can only detect the mm/submm dust continuum emission but it can not observe the specific grain and ice spectral features that are needed to determine the dust composition and its formation history along the life-cycle of interstellar matter.

- ALMA will not even touch several aspects of the chemical complexity in the Universe. In particular, it will not observe molecules without permanent electric dipole or the mid-IR emission of PAHs, thus missing a significant fraction of the organic chemistry in Space.

Note, however, that the above IR spectral diagnostics become available to ALMA in highly-redshifted extragalactic sources. In particular, ALMA will detect the restframe mid- and far-IR spectrum of sources at redshifts of $z>\frac{315}{\lambda_{\text {rest }}(\mu \mathrm{m})}-1$. For example, $z>4$ for the $[\mathrm{O} \mathrm{I}] 63 \mu \mathrm{m}$ line and $z>10$ for the $\mathrm{H}_{2} 0-0 S(0)$ line at $\lambda_{\text {rest }}=28 \mu \mathrm{m}$. 


\section{EXAMPLES OF MID-IR AND FAR-IR SPECTROSCOPY}

We now review some interesting results of previous midand far-IR observations towards Sgr B2 and Orion KL high-mass star forming regions (SFRs). Sgr B2 ( $28.5 \mathrm{kpc}$ ) and Orion KL ( $\simeq 450 \mathrm{pc}$ ) can be considered as two of the most remarkable giant molecular clouds for astrochemistry studies. They are also very appropriate sources to construct a template for more distant (e.g., fainter) and unresolved regions (e.g., extragalactic). The cores of both SFRs are the most prolific sites of molecular line emission/absorption in the Galaxy (in terms of density and intensity/absorption depth of lines). Due to the large column density of warm molecular material (Sgr B2) or due to its proximity (Orion), both complexes are among the brightest far-IR sources of the sky. For these reasons, Sgr B2 and Orion KL were fully surveyed between $\sim 2.4$ and $197 \mu \mathrm{m}$ by ISO's SWS and LWS spectrometers. The resulting spectra remain unique examples of the diagnostic power of this domain, and are archetypes of what SPICA could routinely observe in much fainter sources (protoplanetary disks, outer Solar System bodies, etc.).

\subsection{The MID- AND FAR-IR SPECTRUM OF A SFR}

The far-IR spectrum (47-196 $\mu \mathrm{m}$ ) of Sgr B2 (see Figure 2; Goicoechea et al. 2004; Polehampton et al. 2007 and references therein) contains: (i) The peak of the thermal emission from dust (a blackbody at $\sim 30 \mathrm{~K}$ roughly peaks at $\sim 100 \mu \mathrm{m}$ ); (ii) Atomic fine structure lines that are major coolants of the warm gas, and excellent discriminants of the PDR, HiI and shock emission ([O I], [O III], [C II], [N II], [N III]); (iii) Rotational lines from key light hydrides $\left(\mathrm{H}_{2} \mathrm{O}, \mathrm{OH}, \mathrm{H}_{3} \mathrm{O}^{+}, \mathrm{CH}_{2}, \mathrm{CH}, \mathrm{NH}_{3}, \mathrm{NH}_{2}, \mathrm{NH}, \mathrm{HF}, \mathrm{HD}\right.$, $\mathrm{H}_{2} \mathrm{D}^{+}$) and ro-vibrational lines from abundant nonpolar molecules $\left(\mathrm{C}_{3}\right)$. These species provide unique information on the prevailing physical conditions and on the basic $\mathrm{O}, \mathrm{C}, \mathrm{N}$ and D chemistry. Most of the molecular lines appear in absorption whereas atomic and ionic lines appear in emission (except for absorption in the [O I]63 and [C II] $158 \mu \mathrm{m}$ lines). In particular, [O I] 63 and [C II] $158 \mu \mathrm{m}$ absorption line profiles provide clues regarding several peculiarities observed in some extragalactic spectra at lower resolution (e.g., the $[\mathrm{CII}] /$ far-IR deficit). The main gas component traced by far-IR observations is the warm, low density envelope of Sgr B2 $\left(\mathrm{T}_{k} \simeq 300 \mathrm{~K} ; n\left(\mathrm{H}_{2}\right)<10^{4} \mathrm{~cm}^{-3}\right)$. Given the low densities in this component, no high- $J$ CO line was detected at ISO's sensitivity. This situation may apply to other warm regions observed in the far-IR. The warm, low-density gas is particularly difficult to trace in the millimeter domain where one usually observes molecular emission lines from collisionally excited gas. Finally, because of its location in the Galactic Center, all groundstate lines towards Sgr B2 show a broad absorption profile ( $\Delta v \simeq 200 \mathrm{~km} \mathrm{~s}^{-1}$ ) due to foreground absorption produced by the spiral arm diffuse clouds in the line of sight.
On the other hand, the far-IR spectrum of Orion KL (Lerate et al. 2006 and references therein) is dominated by emission lines from molecular $\left(\mathrm{H}_{2} \mathrm{O}\right.$, high- $J \mathrm{CO}, \mathrm{OH}$, $\mathrm{NH}_{3}$ ) and atomic species. Interestingly enough, $\mathrm{H}_{2} \mathrm{O}$ and $\mathrm{OH}$ line profiles show a complex behavior evolving from pure absorption, P Cygni type, to pure emission, depending on the transition wavelength, $E_{u p}$ and line opacity. These lines arise from Orion outflow(s) and associated shocked regions. Without resolving these profiles, low resolution spectra of similar regions may lead to a misinterpretation of the prevailing dynamics and physical conditions. Given the high densities and temperatures of Orion's shocked gas, CO pure rotational emission up to $J=39$ has been detected (with an upper energy level of $\mathrm{E}_{u p} / k \simeq 4,000 \mathrm{~K} !$ !).

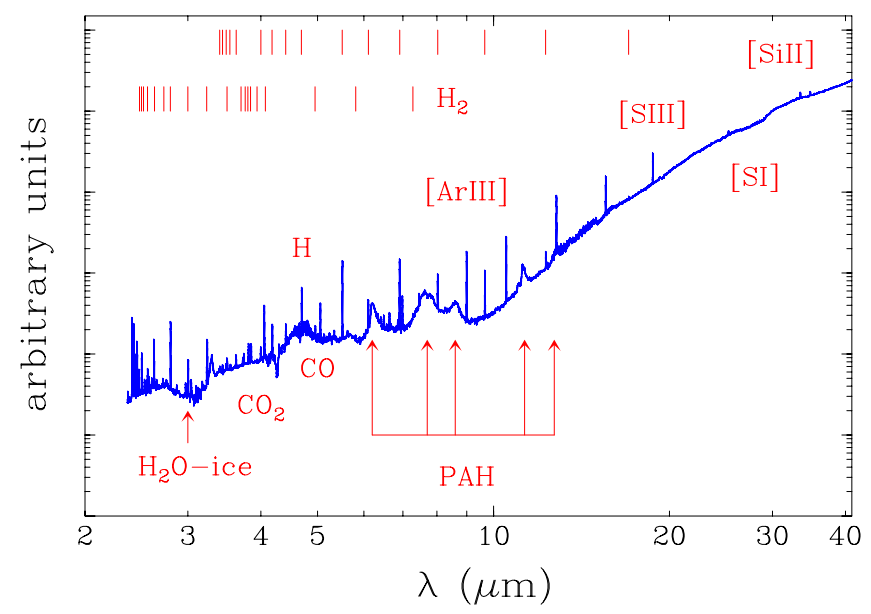

Figure 3. Mid-infrared spectrum of Orion Peak 1 shock obtained in the ISO/SWS grating scan observing mode $(R \sim 2,000)$. Some of the strongest lines and bands are labelled. The continuum levels of the individual bands, which differ due to aperture changes, were adjusted to make the spectrum appear continuous. Adapted from Rosenthal et al. (2000).

Fig. 3 shows the ISO/SWS $(2.4-45 \mu \mathrm{m})$ spectrum of the brightest mid-IR $\mathrm{H}_{2}$ emission peak of the Orion outflow (see Rosenthal et al. 2000). This is one of the most spectacular shocked regions in the galaxy. $56 \mathrm{H}_{2}$ ro-vibrational (up to $E_{u} / k=14,000 \mathrm{~K}$ ) and pure rotational lines from the $\mathrm{H}_{2}$ 0-0 $S(1)$ to 0-0 $S(25)$ lines are detected. The spectrum also shows a number of $\mathrm{PAH}$ bands, $\mathrm{H}$ I recombination lines, ro-vibrational lines of $\mathrm{CO}(\nu=1-0 ; \sim 4.7 \mu \mathrm{m})$ and $\mathrm{H}_{2} \mathrm{O}\left(\nu_{2}=1-0 ; \sim 6.5 \mu \mathrm{m}\right)$, and many ionic fine structure lines ([Ne III] 36, [Fe II] 36, [Si II] 35, [S III] 33, [Fe II] 26, [S I] 25, [Fe III $] 23$, [Ar III]22,[S III]19, [Fe II]18, [P III]18, [Ne III]16, [Ne II $] 13,[\mathrm{~S}$ IV]11, [Ar III] 9 , [Ar II] 7 and $[\mathrm{Ni}$ II $] 7 \mu \mathrm{m})$, most of them coming from the foreground HII region and its bounding PDR. Van Dishoeck et al. (1998) also observed the Orion-IRc2 position with the same spectrometer. In addition, several broadband features associated with silicate grains and $\mathrm{H}_{2} \mathrm{O}$-ice and $\mathrm{CO}_{2}$-ice were detected along the line of sight to the strong mid-IR continuum of IRc2. 


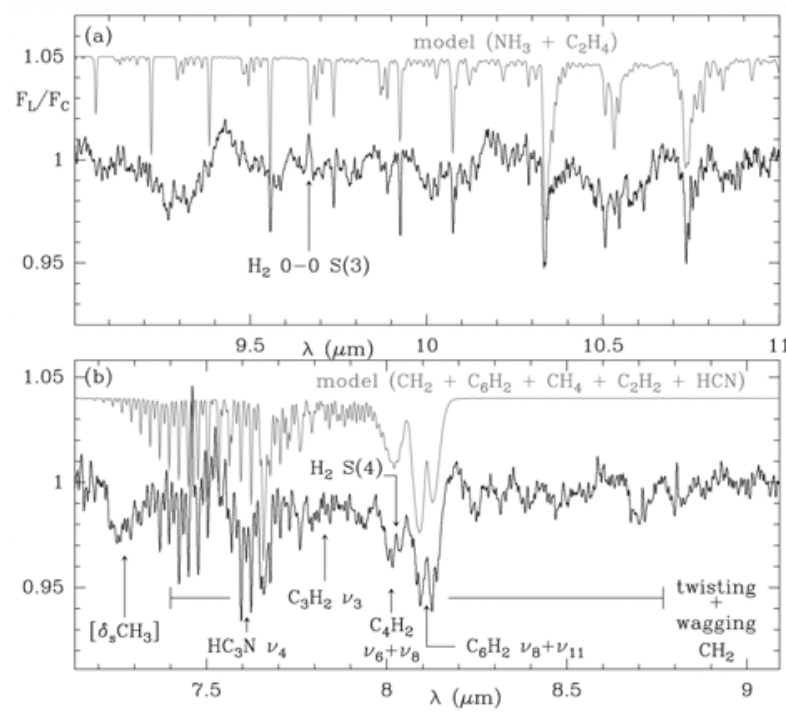

Figure 4. Mid-IR spectrum of CRL 618 at selected wavelengths. The thin line in each panel corresponds to the model results discussed by Cernicharo et al. (2001a). The feature at 7.27 $\mu \mathrm{m}$ corresponds to the symmetric bending mode of $\mathrm{CH}_{3}$. The broad range of absorption bands produced by wagging and twisting modes of $\mathrm{CH}_{2}$ is indicated by the horizontal line (Silverstein 8 Webster 1998). Figure from Cernicharo et al. (2001a).

\subsection{Mid-IR: Chemical COMPleXity}

Mid-IR spectroscopy (vibrational spectroscopy in particular) is a powerful tool to reveal the chemical complexity in the Universe. A remarkable example is the organic composition of the circumstellar envelopes around evolved stars. Many organic species lack rotational spectra to be observed from radiotelescopes (e.g., polyacetylenic chains; see Figure 4). Benzene, the most basic aromatic unit, has been detected outside the Solar System only towards CRL 618 carbon-rich protoplanetary nebula, PPNe (see Figure 5; Cernicharo et al. 2001b) where the physical conditions in the inner CSE (shocks and UV photons) seem to drive the polymerization of acetylene and the formation of benzene. This means that carbon-rich PPNes are probably the best organic chemistry factories in space and much can be learned about the gas/dust chemistry interplay (e.g., the formation of larger aromatic species or PAHs). SPICA's very high-resolution mid-IR spectrometers will have the required sensitivity to study the organic content and reveal the degree of chemical complexity, not only towards the brightest sources in the sky, but everywhere in the Milky Way and in nearby galaxies.

\section{Conclusions}

The mid- and far-IR domains provides key spectral diagnostics of the gas phase chemistry (e.g., water vapor, $\mathrm{H}_{2}$, PAHs, organic species), of the prevailing physical conditions (atomic and ionic fine structure lines), and of the

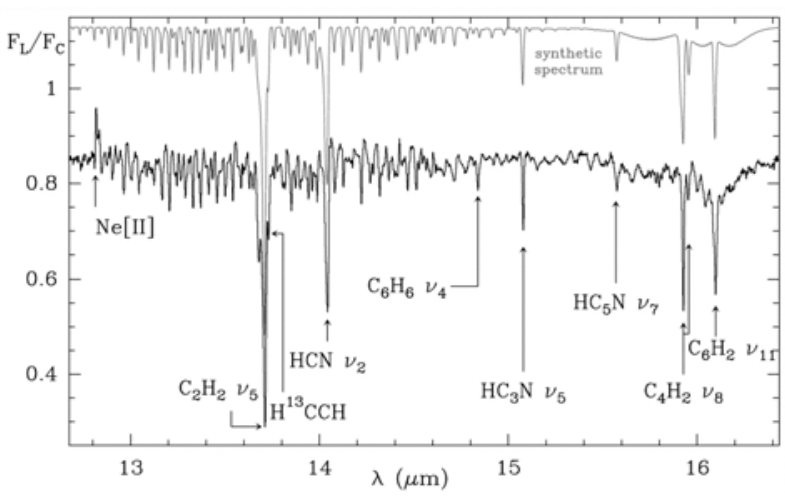

Figure 5. Mid-IR spectrum of CRL 618 around $15 \mu \mathrm{m}$. The infrared bands of the polyynes $\left(\mathrm{C}_{4} \mathrm{H}_{2}, \mathrm{C}_{6} \mathrm{H}_{2}\right)$, benzene, and cyanopolyynes are indicated by arrows. The thin line corresponds to the synthetic spectrum for $\mathrm{C}_{2} \mathrm{H}_{2}, \mathrm{HCN}, \mathrm{C}_{4} \mathrm{H}_{2}, \mathrm{C}_{6} \mathrm{H}_{2}$, $H_{3} N$, and $H_{5} N$. All the narrow features to the right and left of the Q-branches of $\mathrm{C}_{2} \mathrm{H}_{2}$ and $\mathrm{HCN}$ correspond to the individual rovibrational lines of the $P$ - and $R$-branches of these same two species. Figure from Cernicharo et al. (2001b).

dust mineral/ice composition that can not be observed at other wavelengths. Thanks to SPICA, uninterrupted studies in the critical $\sim 5-210 \mu \mathrm{m}$ range will be possible since the launch of ISO with orders of magnitude higher sensitivity and resolutions. Therefore, SPICA observations will revolutionize the study the life-cycle of interstellar matter in nearby galaxies the same way ISO revolutionized the study of the ISM in the Milky Way in the late 90's.

\section{ACKNOWLEDGEMENTS}

JRG thanks the European and Japanese SPICA science teams for fruitful discussions and different contributions to develop the "gas and dust life-cycle" case. We warmly thank Marcelino Agúndez for letting use his "life-cycle figure". JRG is supported by a Ramón y Cajal research contract.

\section{REFERENCES}

Agúndez, M. 2009, Ph. D. Thesis, UAM.

Cernicharo, J. et al. 2001a, ApJ, 546, L127.

Cernicharo, J. et al. 2001b, ApJ, 546, L123.

Cernicharo, J. \& Crovisier, J. 2005, SSR, 119, 1-4, 29-69.

Goicoechea, J.R. et al. 2004, ApJ, 600, 214.

Goicoechea, J.R. et al. 2005, ApJ, 619, 29.

González-Alfonso, E. et al. 2004, ApJ, 613, 247.

Kessler, M. F. et al. 1996, $A \& A, 315, \mathrm{~L} 27$.

Murakami, H. et al. 2007, PASJ, 59S, 36.

Okada, Y. et al. 2008, ApJ, 682, 416.

Pilbratt, G. L. 2009, EAS Publications Series, 3, 15-31.

Polehampton, E.T., et al. , 2007, MNRAS, 377, 1122.

Rosenthal, D. et al. 2000, $A \mathscr{E} A, 356,705$

van Dishoeck, E.F. et al. 1998, ApJ, 502, L173.

van Dishoeck, E.F. 2004, ARA\&A, 42, 119.

Werner, M. W. et al. 2004, ApJS, 154, 1. 